\title{
MicroRNA-378 enhances migration and invasion in cervical cancer by directly targeting autophagy-related protein 12
}

\author{
DONGMEI TAN ${ }^{1,2^{*}}$, CHAO ZHOU ${ }^{1,3^{*}}$, SAI HAN ${ }^{1}$, XUETAO HOU ${ }^{2}$, SHUFANG KANG ${ }^{2}$ and YOUZHONG ZHANG \\ ${ }^{1}$ Department of Obstetrics and Gynecology, Qilu Hospital of Shandong University, Jinan, Shandong 250012; \\ ${ }^{2}$ Department of Obstetrics and Gynecology, Laiwu Maternal and Child Health Care Hospital, Laiwu, Shandong 271100; \\ ${ }^{3}$ Department of Obstetrics and Gynecology, Binzhou Medical University Hospital, Binzhou, Shandong 256603, P.R. China
}

Received July 12, 2017; Accepted January 8, 2018

DOI: $10.3892 / \mathrm{mmr} .2018 .8645$

\begin{abstract}
Cervical cancer is the second most common type of cancer among women worldwide and a leading cause of mortality in women. Metastases reduce the overall survival rate in patients with cervical cancer. Thus, it is clinically urgent to investigate the molecular mechanism of cervical cancer metastasis. The aim of the present study was to investigate the mechanism of microRNA (miR)-378 in the metastasis of cervical cancer. In the present study, miR-378 expression levels were significantly upregulated in cervical cancer tissues and cervical intraepithelial neoplasia III tissues when compared with normal cervix tissues. Re-expression of miR-378 significantly promoted tumor migration and invasion in vitro, and metastasis in vivo, while downregulation of miR-378 suppressed the effect in vitro. Luciferase reporter assay revealed that autophagy-related protein 12 (ATG12) was a direct target of miR-378 and its expression was downregulated by miR-378. In cervical cancer tissues with lymph node metastasis, miR-378 was upregulated while ATG12 was downregulated when compared with lymph node negative cases. To the best of our knowledge, the present study is the first to provide evidence that miR-378 may be associated with ATG12. Collectively, the data of the present study suggested that miR-378 may function as an oncogene by promoting metastasis in cervical cancer. The finding that miR-378 targets ATG12 indicated that miR-378 may have a potential role in autophagy. These findings may provide novel insights into the mechanism of metastasis in cervical cancer and a novel therapeutic target for the treatment of cervical cancer.
\end{abstract}

Correspondence to: Dr Youzhong Zhang, Department of Obstetrics and Gynecology, Qilu Hospital of Shandong University, 107 Wenhua Road, Jinan, Shandong 250012, P.R. China

E-mail: zhangyouzhong@sdu.edu.cn

*Contributed equally

Key words: microRNA-378, cervical cancer, migration, invasion, autophagy-related protein 12

\section{Introduction}

Cervical cancer is the second most common cancer among women worldwide and a leading cause of mortality in women (1). The most important risk factor is infection with oncogenic high-risk human papillomaviruses (HPVs), including HPV16 and HPV18 (2). Developments in the diagnosis and treatment in cervical cancer have been observed; however, the five-year survival rate is $\sim 65 \%$ in patients with lymph node metastases (3). Thus, it is clinically important to investigate the molecular mechanism of metastasis of cervical cancer.

MicroRNAs (miRNAs/miRs) are small non-coding RNA sequences with $\sim 18-25$ nucleotides that modulate translational efficiency or stability by targeting the 3'-untranslated region (3'-UTR) of mRNA. Increasing evidence has demonstrated that miRNAs serve a key role in the progression and oncogenesis of a variety of cancers, including cervical cancer (4), gastric cancer (5), lung cancer (6) and breast cancer (7). In cervical cancer, numerous miRNAs were demonstrated to be involved in cancer initiation, promotion and progression. miR-506 acts as a tumor suppressor by inhibiting cervical cancer growth (8). miR-100 is downregulated in cervical cancer and may regulate cell proliferation, cycle and apoptosis (9). miR-424 functions as a tumor suppressor by inhibiting cell proliferation, migration and invasion of cervical cancer (10). In addition, Wang et al (4) reported that miR-378 was aberrantly upregulated in HPV16/18-positive cervical cancer and the increase of miR-378 may also be induced by oncoprotein E6 and/or E7; however, the detailed mechanism of miR-378 in cervical cancer requires further investigation.

Autophagy-related protein 12 (ATG12) is a member of the ATG family associated with autophagy. Numerous core ATGs in two ubiquitin-like conjugation systems are essential for autophagosome formation. In the first system, ATG12 is activated by ATG7, transferred to ATG10 and ultimately attached to ATG5 (11). In the second system, microtubule-associated protein 1 light chain 3 conjugates to lipid phosphatidylethamine by ATG7 and ATG3. In addition to ATG5, a recent study demonstrated that ATG12 also conjugates to ATG3 (12). The ATG12-ATG3 complex promotes autolysosome formation under nutrient-rich conditions and ATG12-ATG3 interacts with apoptosis-linked gene 2-interacting protein $\mathrm{X}$ to promote 
basal autophagic flux and late endosome function (12). Thus, these previous studies indicated that ATG12 may serve a key role in autophagy; however, the association between miR-378 and ATG12 remains to be investigated.

In the present study, miRNA-378 expression levels were upregulated in cervical intraepithelial neoplasia (CIN) III and cervical cancer tissues. Upregulation of the miRNA-378 expression levels significantly promoted cervical cancer migration and invasion in vitro and in vivo. Furthermore, ATG12 was identified as a functional and direct target of miR-378. These findings may provide novel insights into the molecular mechanism of metastasis in cervical cancer.

\section{Materials and methods}

Tissues and cell lines. A total of 185 female patients (Table I) were involved in the present study between January 2012 to January 2016 at the Department of Gynecology, Qilu Hospital of Shandong University (Shandong, China). In total, 60 normal cervix tissues were collected from patients who underwent a hysterectomy due to benign gynecological diseases. Additionally, 70 CIN III tissues were obtained from patients who received cold-knife conization surgery and 55 cervical cancer tissues were collected from patients who underwent radical hysterectomy and were diagnosed with squamous cervical carcinoma. Tissues were frozen in liquid nitrogen and stored at $-80^{\circ} \mathrm{C}$. Written informed content was obtained from all patients and the present study was approved by the Ethics Committee of Qilu Hospital of Shandong University.

The 2 cervical cancer cell lines, HeLa and C-33A, were employed in the present study. HeLa and C-33A cells were cultured in RPMI-1640 medium (Gibco; Thermo Fisher Scientific, Inc., Waltham, MA, USA) with $10 \%$ fetal bovine serum (FBS; HyClone; GE Healthcare Life Sciences, Logan, UT, USA) and $1 \%$ penicillin/streptomycin. All cells were incubated in a humidified incubator with $5 \% \mathrm{CO}_{2}$ at $37^{\circ} \mathrm{C}$.

Oligonucleotide construction and transfection. To investigate the biological function of miR-378 in cell migration and invasion, GIPZ-miR-378 was transfected into C-33A cells to overexpress miR-378, and the miR-378 inhibitor was transfected into HeLa cells to inhibit miR-378. Oligonucleotides including miR-378 mimics (forward, 5'-ACUGGACUUGGA GUCAGAAGG-3' and reverse, 5'-UUCUGACUCCAAGUC CAGUUU-3'), miR-378 inhibitor (5'-CCUUCUGACUCC AAGUCCAGU-3') and mimic negative controls (forward, 5'-UUCUCCGAACGUGUCACGUTT-3' and reverse, 5'-ACG UGACACGUUCGGAGAATT-3'); inhibitor negative control (5'-CAGUACUUUUGUGUAGUACAA-3') were purchased from Dharmacon (Lafayette, CO, USA). RNA oligonucleotides were transfected at a final concentration of $50 \mathrm{nM}$ using Lipofectamine 2000 (Invitrogen, Carlsbad, California, USA) according to the manufacturer's protocol. Small interfering (si)RNA-ATG12 and scrambled negative control were supplied by Shanghai GenePharma Co., Ltd. (Shanghai, China). The sequences of the siRNA-ATG12 and negative control are as follows: siRNA-ATG12 (forward, 5'-GUUGCAGCUUCCUAC UUCATT-3'; reverse, 5'-UGAAGUAGGAAGCUGAACTT-3'), scrambled negative control siRNA was 5'-ACTACCGTT GTTATAGGTG-3'. The miR-378 overexpression construct
(GIPZ-miR-378) and control (GIPZ-NC) were provided by Guangzhou Ribobio Co., Ltd. (Guangzhou, China). The multiplicity of infection (MOI) of HeLa and C-33A cell was tested at 10, 20,40 and 80 using lentiviral transfection. The optimum MOI of HeLa was 10 and MOI of C-33A was 20. Subsequent experiments were performed $48 \mathrm{~h}$ after infection.

Reverse transcription-quantitative polymerase chain reaction $(R T-q P C R)$. Total RNA was extracted from cells or tissues using TRIzol reagents (Invitrogen; Thermo Fisher Scientific, Inc.) according to the manufacturer's protocols. Reverse-transcribed complementary DNA was synthesized at $37^{\circ} \mathrm{C}$ for $15 \mathrm{~min}$ and $80^{\circ} \mathrm{C}$ for $5 \mathrm{sec}$ using a PrimeScript RT reagent kit (Takara Biotechnology, Co., Ltd., Dalian, China). Quantitative real-time PCR was performed with StepOne plus (Applied Biosystems, Foster City, CA, USA) using SYBR Premix ExTaq (Takara Biotechnology, Dalian, China). For miR-378 detection, U6 was used as an internal reference of miRNA. GAPDH was used as an internal reference of mRNA. The following primers were used: ATG12 (forward, 5'-CTG CTGGCGACACCAAGAAA; reverse 5'-CGTGTTCGCTCT ACTGCCC-3'), miR-378 (forward, 5'-GGGACTGGACTT GGAGTCA-3'; reverse, 5'-GTGCGTGTCGTGGAGTCG-3'), U6 (forward 5'-CTCGCTTCGGCAGCACA-3'; reverse, 5'-AACGCTTCACGAATTTGCGT-3'), GAPDH (forward, 5'-GGAGCGAGATCCCTCCAAAAT-3'; reverse 5'-GGC TGTTGTCATACTTCTCATGG-3').

The following thermocycling conditions were used for detection of mRNAs: Initial denaturation at $95^{\circ} \mathrm{C}$ for $5 \mathrm{sec}$; 40 cycles of $95^{\circ} \mathrm{C}$ for $30 \mathrm{sec}, 62^{\circ} \mathrm{C}$ for $30 \mathrm{sec}$ and $72^{\circ} \mathrm{C}$ for $30 \mathrm{sec}$. The following thermocycling conditions were used for detection of miRs: Initial denaturation at $95^{\circ} \mathrm{C}$ for $15 \mathrm{sec}$, 40 cycles of $94^{\circ} \mathrm{C}$ for $15 \mathrm{sec}, 55^{\circ} \mathrm{C}$ for $30 \mathrm{sec}$ and $70^{\circ} \mathrm{C}$ for $30 \mathrm{sec}$. All reactions were run in triplicate. The $2^{-\Delta \Delta \mathrm{Cq}}$ method was used for quantification (13).

Western blot analysis. A total of $5 \times 10^{6} \mathrm{HeLa}$ cells and $8 \times 10^{6} \mathrm{C}-33 \mathrm{~A}$ cells were harvested and lysed using radioimmunoprecipitation assay lysis buffer (Beyotime Institute of Biotechnology, Shanghai, China). The concentration of cell protein was measured with a Bicinchoninic Acid Protein Assay kit (Bio-Rad Laboratories, Inc., Hercules CA, USA). A total of $30 \mu \mathrm{g} /$ lane whole cell protein was separated by $10 \%$ SDS-PAGE and blotted onto nitrocellulose membranes (EMD Millipore, Billerica, MA, USA) and blocked in Tris-buffered saline containing $0.1 \%$ Tween-20 with $5 \%$ non-fat milk at room temperature for $1 \mathrm{~h}$. The membranes were then incubated with the following primary antibodies: CCND1 (1:2,000; cat. no. ab21699; Abcam, Cambridge, MA, USA), phosphorylated-Rb (Ser608; 1:5,000; cat. no. 8145S), ATG12 (1:2,000; cat. no. 4180T) (both from Cell Signaling Technology, Inc., Danvers, MA, USA) and $\beta$-actin (1:10,000, cat. no. ab49900; Abcam) at $4^{\circ} \mathrm{C}$ overnight, followed by incubation with the horseradish peroxidase(HRP) conjugated secondary antibodies (anti-mouse IgG; 1:10,000; cat. no. 7076; and anti-rabbit IgG; 1:10,000; cat. no. 7074; both from Cell Signaling Technology, Inc.) at room temperature for $2 \mathrm{~h}$. Protein bands were visualized using the SuperSignal West Pico chemiluminescent substrate (Pierce; Thermo Fisher Scientific, Inc.). Protein band intensity was quantified by 
Table I. Main characteristics of patients enrolled in the present study.

\begin{tabular}{lccc}
\hline Characteristic & Squamous carcinoma of cervix & CIN III & N \\
\hline Cases (n) & 55 & 70 & 60 \\
Age (years) & $44.2 \pm 7.2$ & $39.6 \pm 7.3$ & $48.8 \pm 5.6$ \\
Surgery received & Radical hysterectomy & CKC & NA \\
Stage & IB-IIA & NA & NA \\
Lymph node metastasis (n) & 17 & NA & NA \\
Positive & 38 & NA \\
Negative & & & \\
\hline
\end{tabular}

Age values are presented as the mean \pm standard deviation. CIN III, cervical intraepithelial neoplasia III; N, normal cervix; CKC, cold-knife conization; NA, not available.

using the ImageJ (version 1.6; National Institutes of Health, Bethesda, MD, USA).

Luciferase reporter assay. To investigate the downstream targets of miR-378, putative targets were analyzed using TargetScan (release 3.1; www.targetscan.org/mamm_31/). For the luciferase reporter assay, the pmirGLO dual-luciferase miRNA target expression vector (Promega Corporation, Madison, WI, USA) was employed in the present study to demonstrate the target of miR-378. Using an Effectene transfection reagent according to the manufacturer's protocol (Qiagen GmbH, Hilden, Germany), HeLa and C-33A cells were co-transfected with pmirGLO-ATG12-3'-UTR-wild-type (WT) or pmirGLO-ATG12-3'-UTR-mutant (MUT) plus miR-378 mimic or negative control. MUT 3'-UTR was generated using QuikChange Site-Directed Mutagenesis kit (Stratagene; Agilent Technologies, Inc., Santa Clara, CA, USA). Luciferase activity was detected $48 \mathrm{~h}$ post-cell transfection using the GloMax fluorescence reader (Promega Corporation). Renilla luciferase was used as the internal control.

Immunohistochemistry (IHC) assay. Tissues were fixed in 4\% paraformaldehyde for $24 \mathrm{~h}$ at $4^{\circ} \mathrm{C}$, paraffin embedded and cut into $4 \mu \mathrm{m}$ sections. Slides were deparaffinized and rehydrated using xylene and descending alcohol series. Antigen retrieval was performed at $95^{\circ} \mathrm{C}$ for $5 \mathrm{~min}$ in sodium citrate buffer (10 mM sodium citrate; $0.05 \%$ Tween-20; pH 6.0) and washed 3 times for 2 min with PBS.Then slides were blocked with $3 \%$ hydrogen peroxidase in methanol and $10 \%$ goat serum (Beijing Solarbio Science \& Technology Co., Ltd., Beijing, China) for $2 \mathrm{~h}$ at room temperature. Slides were incubated with ATG12 antibody (1:500; cat. no. NBP2-15501, Novus Biologicals, LLC, Littleton, CO, USA) overnight at $4^{\circ} \mathrm{C}$ and then with HRP-labeled goat anti-rabbit IgG (1:50; cat. no. A0208; Beyotime Institute of Biotechnology) antibody for $2 \mathrm{~h}$ at room temperature. Streptavidin-horseradish peroxidase was added, and sections were stained with 3,3'-diaminobenzidine(DAB) substrate for $3 \mathrm{~min}$ and counterstained with hematoxylin $30 \mathrm{sec}$ at room temperature. For blank controls, the primary antibodies were replaced with PBS solution (100 mM; pH 7.4). All slides were evaluated by two independent investigators without prior knowledge of the clinical data of the specimens. According to staining intensity, specimens were scored as absent (0), weak (1), moderate (2) or intense (3). The tissues with score 0 and 1 were defined as negatively stained, while 2 and 3 defined positive staining of ATG12. The slides were analyzed and imaged using a bright-field microscope (Olympus BX50; Olympus Corporation, Tokyo, Japan).

Cell migration and invasion. Cell migration and invasion potential was evaluated using Transwell inserts $(8 \mu \mathrm{m}$ pores; BD Biosciences, San Jose, CA, USA). All the experiments were repeated 3 times. C-33A/HeLa cells transfected with si-ATG12, GIPZ-miR-378 and miR-378 inhibitor were pre-cultured in RPMI-1640 medium without FBS at $37^{\circ} \mathrm{C}$ for $24 \mathrm{~h}$ in 12 -well plates. For the migration assay, $1 \times 10^{5}$ cells were placed in the top chamber of Transwell plates and incubated at $37^{\circ} \mathrm{C}$ for $24 \mathrm{~h}$ with serum-free RPMI-1640 medium. For the invasion assay, $1 \times 10^{5}$ cells were placed in the top chamber of Transwell plates, which were pre-coated with extracellular matrix gel (Matrigel) (BD Biosciences) and incubated at $37^{\circ} \mathrm{C}$ for $24 \mathrm{~h}$ with serum-free medium; RPMI-1640 medium with 15\% FBS (HyClone; GE Healthcare Life Sciences) was added to the lower chamber as a chemoattractant. Following $48 \mathrm{~h}$ incubation, cells remaining on the upper surface were wiped off using cotton swabs and cells on the lower surface were fixed in $4 \%$ paraformaldehyde for $30 \mathrm{~min}$ at room temperature and stained with $0.1 \%$ crystal violet for $30 \mathrm{~min}$ at room temperature, and counted under a light microscope (magnification, x100) in five random visual fields.

Metastasis assay in vivo. Immunodeficient, 5-week old female BALB/c nu/nu mice (Vital River Laboratory Animal Technology Co., Ltd, Beijing, China) were used for the metastasis assay in vivo. A total of 20 mice (10 in GIPZ-miR-378 group and 10 GIPZ-NC group) were kept under specific pathogen free conditions, 12 -h light/dark cycle at $20-30^{\circ} \mathrm{C}$ and all of mice had free access to food and water. A total of $2 \times 10^{6}$ C-33A cells transfected with GIPZ-miR-378 or GIPZ-NC were injected into mice via a tail vein injection. Following 8 weeks, mice were sacrificed and the lungs were dissected and fixed using $10 \%$ formalin for $24 \mathrm{~h}$ at $4^{\circ} \mathrm{C}$ and paraffin embedded. The paraffin blocks were cut into $4 \mu \mathrm{m}$ thick slices and hematoxylin and eosin staining was performed. Hematoxylin staining was performed for $45 \mathrm{sec}$ and eosin staining for $30 \mathrm{sec}$, both at room temperature. Histological 
A

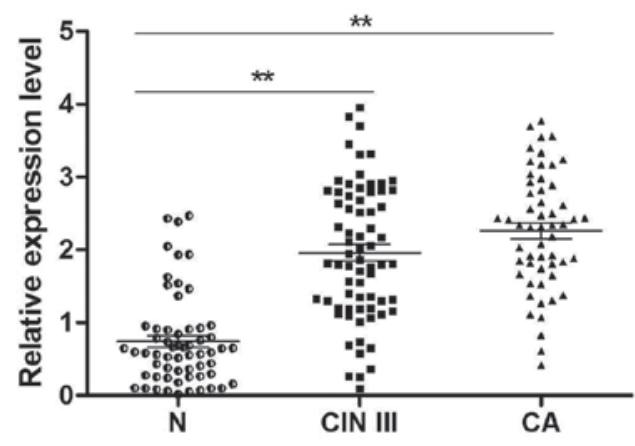

B

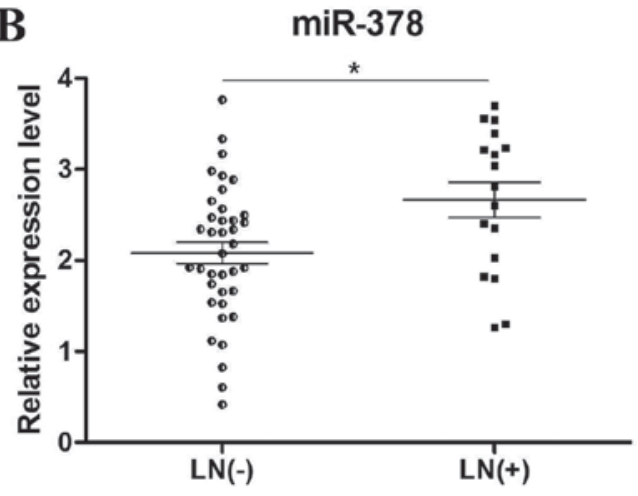

Figure 1. miR-378 was frequently upregulated in CIN III and LN positive CA tissues. (A) miR-378 was significantly upregulated in CA tissues (n=55) and CIN III tissues $(n=70)$ when compared with $\mathrm{N}$ tissues $(\mathrm{n}=60)$. (B) In patients with cervical cancer, the positive group $(\mathrm{n}=17)$ with $\mathrm{LN}$ metastasis exhibited significantly higher expression levels of miR-378 than the negative group $(\mathrm{n}=38)$. Relative expression was normalized to U6. $\mathrm{P}<0.01$ and ${ }^{* * *} \mathrm{P}<0.05$ as indicated. CA, cervical cancer; CIN III, cervical intraepithelial neoplasia III; LN, lymph node; miR, microRNA; N, normal cervix.

A

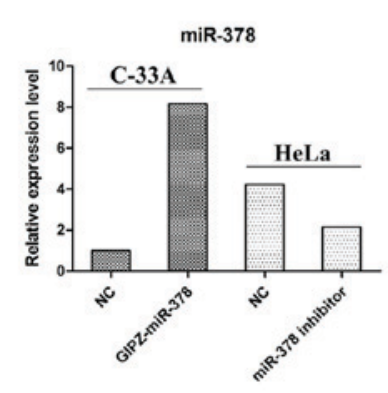

D

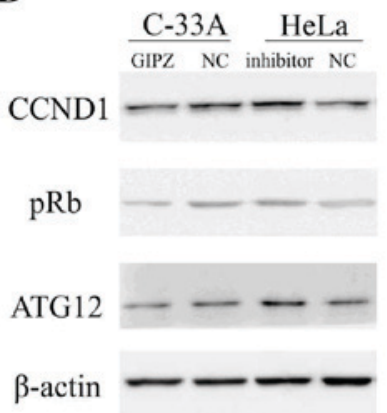

B
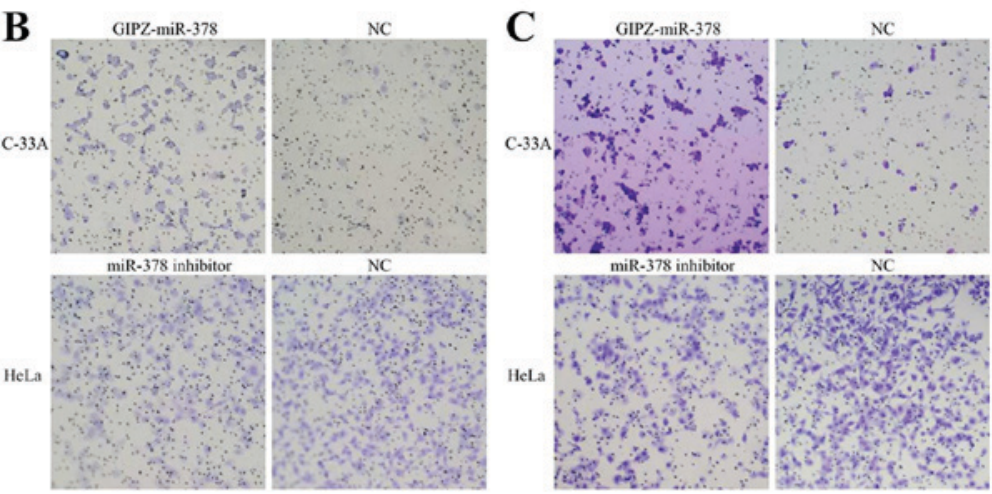

E

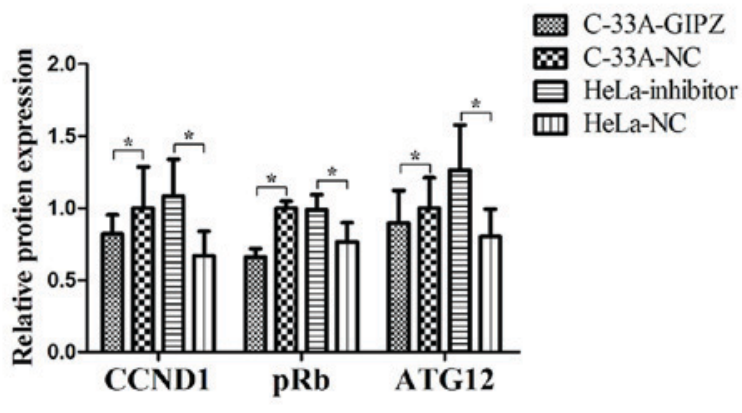

Figure 2. Overexpression of miR-378 promoted cell migration and invasion of cervical cancer. (A) Relative expression of miR-378 was determined by reverse transcription-quantitative polymerase chain reaction. (B) Overexpression in C-33A cells promoted cell migration and knockdown of miR-378 in HeLa cells inhibited cell migration (magnification, x100). (C) Overexpression in C-33A cells promoted cell invasion and knockdown of miR-378 in HeLa cells inhibited cell invasion (magnification, x100). (D) Effects of miR-378 on the protein expression of ATG12 were determined by western blot analysis in C-33A cells transfected with GIPZ-miR-378 and HeLa cells transfected with miR-378 inhibitor. (E) Quantitative analysis of the western blot results in Fig. 2D. Each bar represents the grey area of each band and the statistical significance was analyzed from three independent replicates. The data are presented as the mean \pm standard deviation. ${ }^{*} \mathrm{P}<0.05$. ATG12, autophagy-related protein 12; CCND1, cyclin D1; miR, microRNA; NC, negative control; pRb, retinoblastoma.

examination was confirmed under bright-field microscope. All animal experiments were approved by the Ethics Committee of Qilu Hospital of Shandong University.

Statistical analysis. Statistical analysis was performed using SPSS software (version 19.0; IBM Corp., Armonk, NY, USA). Differences in miR-378 expression levels between groups were examined using one-way analysis of variance, followed by a Tukey's post hoc test. Comparisons in cell density, the relative levels of protein expression and the number of tumor foci between the two different transfection groups were presented as the mean \pm standard deviation from at least three independent experiments and were analyzed using an unpaired Student's t-test. $\mathrm{P}<0.05$ was considered to indicate a statistically significant difference.

\section{Results}

miR-378 is frequently upregulated in CIN III and lymph node positive cervical cancer tissues. Patients enrolled in the 
present study included 3 subgroups, which were normal cervix (N), CIN III and cervical carcinoma (CA). The clinicopathological characteristics of these tissues were listed in Table I. To determine the expression levels of miR-378, RT-qPCR of $\mathrm{N}(\mathrm{n}=60)$, CIN III $(\mathrm{n}=70)$ and cervical cancer tissues $(\mathrm{n}=55)$ was performed. The results revealed that miR-378 expression was significantly increased in CIN III $(\mathrm{P}<0.01)$ and CA tissues $(\mathrm{P}<0.01)$ compared with $\mathrm{N}$ tissues (Fig. 1A). In addition, miR-378 expression levels in lymph node positive $(n=17)$ and negative $(n=38)$ cancer tissues were compared (Fig. 1B), which revealed that the positive group with lymph node metastasis exhibited significantly a higher expression of miR-378 $(\mathrm{P}<0.05)$.

Overexpression of miR-378 significantly promotes cell migration and invasion in vitro. In the two cervical cancer cell lines, miR-378 basic expression levels were detected by RT-qPCR analysis. The successful overexpression or inhibition of miR-378 was determined by RT-qPCR analysis (Fig. 2A). In the Transwell assay, overexpression of miR-378 significantly promoted cell migration and invasion in C-33A cells (Fig. 2B and C), and inhibition of miR-378 significantly suppressed migratory and invasive abilities in HeLa cells (Fig. 2B and C).

In addition, the relative expression levels of ATG12, cyclin D1 (CCND1) and retinoblastoma (pRb) in C-33A cells and HeLa cells were detected (Fig. 2D and E). Overexpression of miR-378 in C-33A cells significantly decreased the expression of ATG12, CCND1 and pRb. In addition, the inhibition of miR-378 in HeLa cells increased the expression levels of ATG12, CCND1 and pRb. These findings indicated that miR-378 may have promoted cell migration and invasion in vitro and regulated the expression of numerous key genes.

ATG12 is a direct target of miR-378 and regulates cell metastasis in cervical cancer. To investigate the downstream targets of miR-378, putative targets were analyzed using TargetScan. One conserved putative binding site was reported at 509-515 bp of ATG12 3'-UTR (Fig. 3A). To verify the putative target, a luciferase reporter assay was performed in C-33A and HeLa cells. When cells were co-transfected with miR-378 mimic and pmirGLO-ATG12-3'-UTR-WT, the relative luciferase activity was significantly decreased compared with the miR-control $(\mathrm{P}<0.01)$ (Fig. 3B). The luciferase activity was rescued when cells were co-transfected with miR-378 mimic and pmirGLO-ATG12-3'-UTR-MUT (Fig. 3B). These findings indicated that ATG12 may be a direct target of miR-378.

To further validate whether ATG12 is downregulated by miR-378 in cancer tissues, the expression of ATG12 in lymph node positive and negative cervical cancer samples was detected by IHC analysis (Fig. 3C). ATG12 expression levels between the two groups were significantly different as they were upregulated in the lymph node negative group (52.9\% positive) compared with in the lymph node positive group (20\% positive; $\mathrm{P}<0.05$; Fig. 3D). Combined with the data presented in Fig. 1B, the results indicated that ATG12 expression levels were inversely associated with miR-378 expression in cervical cancer cases with or without lymph node metastasis.

To evaluate the function of ATG12 in cell metastasis, in vitro migration and invasion assays were conducted using C33-A
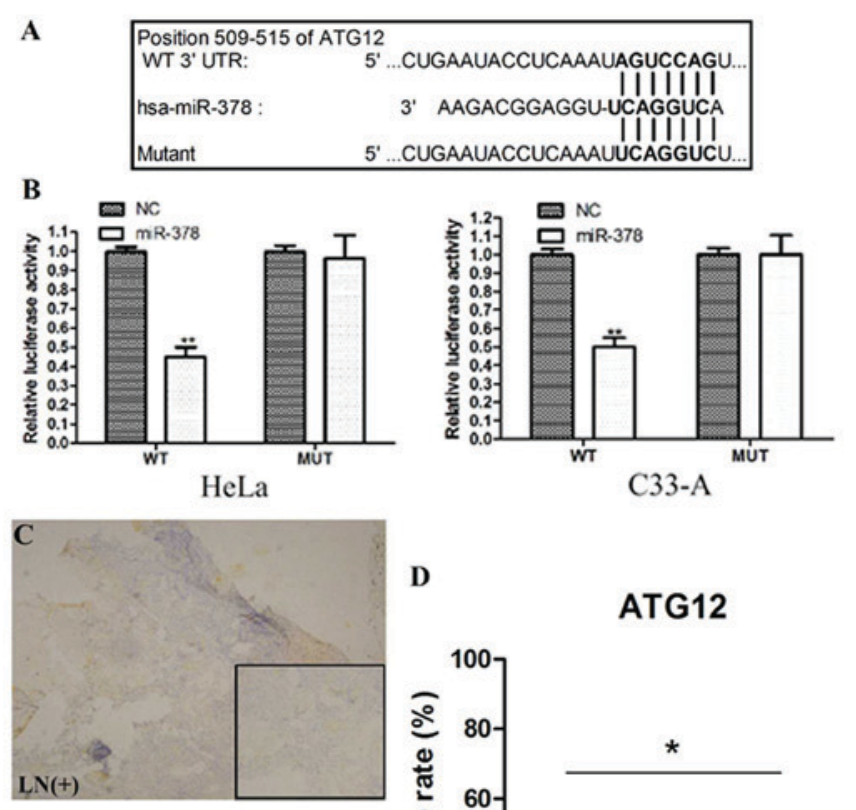

D
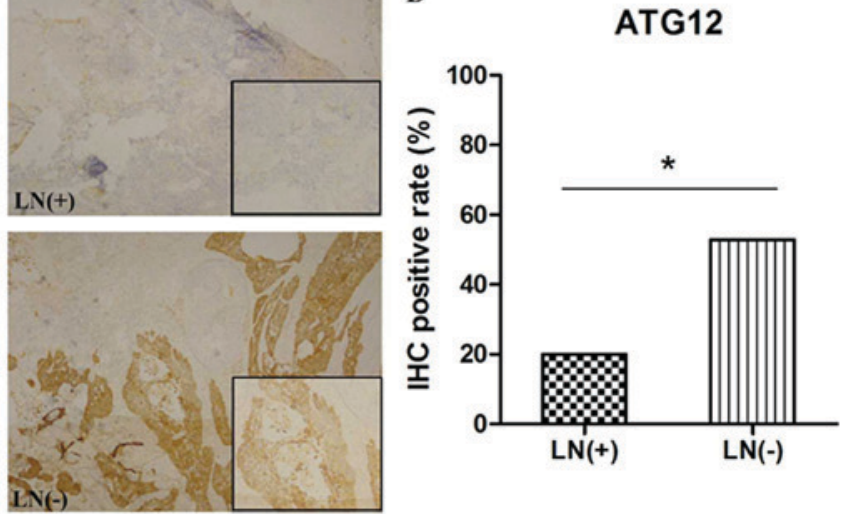

E

Si-ATG12
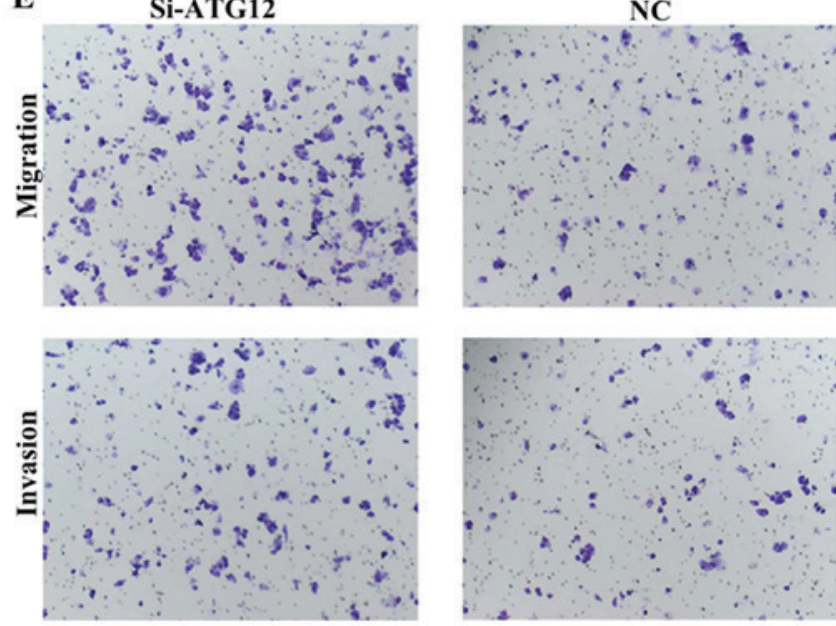

Figure 3. ATG12 was identified as a direct target of miR-378. (A) Putative binding site of miR-378 in the 3'-UTR of ATG12. (B) Luciferase reporter assay in C-33A and HeLa cells. Cells were co-transfected with miR-378 mimics or control plus WT or MUT ATG12 3'-UTR. Firefly luciferase activity was normalized to Renilla luciferase activity. ${ }^{* * *} \mathrm{P}<0.01$ vs. NC. (C) Representative images of ATG12 stain in cervical cancer tissues (magnification, x100). (D) immunohistochemistry staining of the cervical cancer tissues revealed that ATG12 expression was upregulated in LN negative group (52.9\% positive) when compared with the $\mathrm{LN}$ positive group (20\% positive). ${ }^{*} \mathrm{P}<0.05$. (E) Inhibition of ATG12 in C-33A cells promoted cell migration and invasion (magnification, x100). ATG12, autophagy-related protein 12; LN, lymph node; miR, microRNA; MUT, mutant; si, small interfering; UTR, untranslated region; WT, wild-type.

cells. Cells transfected with si-ATG12 exhibited decreased expression of ATG12 mRNA and protein, compared with cells transfected with the negative control (data not shown). Suppression of ATG12 notably promoted cell migration and 

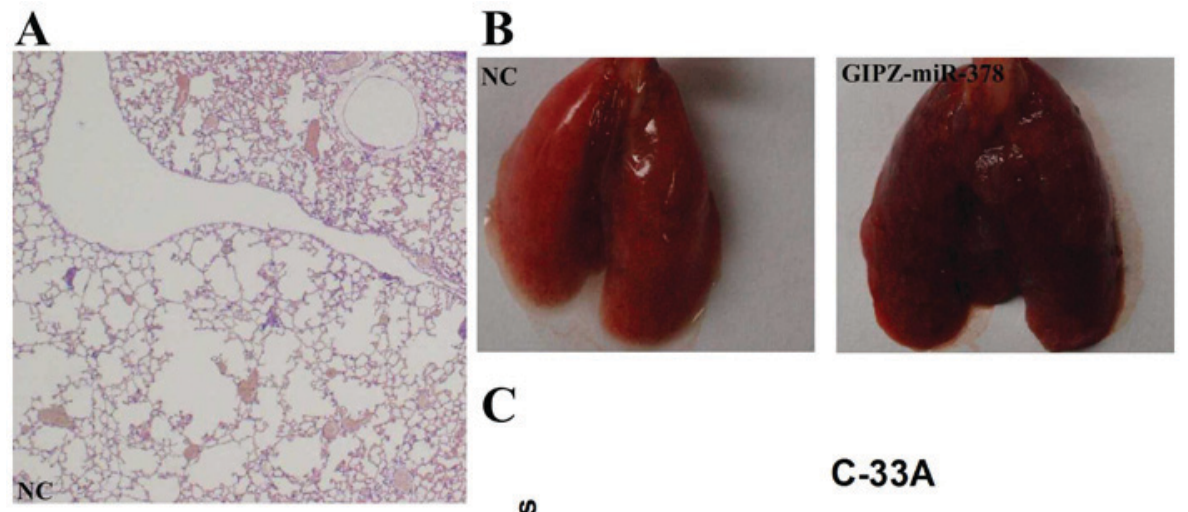

\section{C}
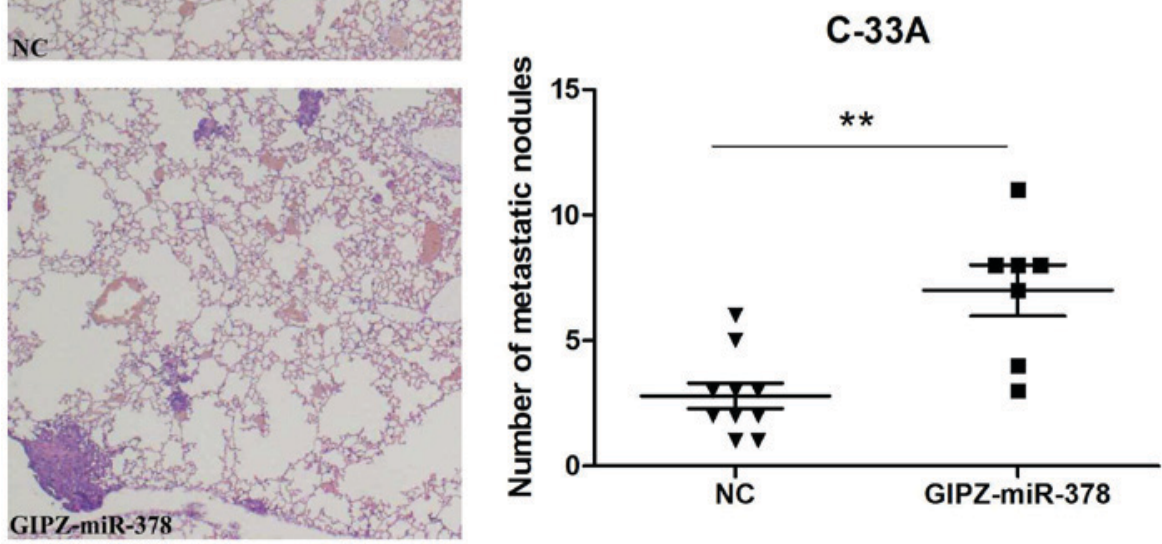

Figure 4. miR-378 promoted cervical cancer metastasis in vivo. (A) Representative hematoxylin and eosin staining sections of distant metastatic nodules in lungs. The upper image is the NC group and the lower image is the GIPZ-miR-378 group. (B) Representative images of lungs at the 8th week post-transfection; the left and right panels present the NC and the GIPZ-miR-378 mouse groups, respectively. (C) The number of distant metastatic nodules in the lung were counted and compared between the control and GIPZ-miR-378 groups. " $\mathrm{P}<0.01$. miR, microRNA; NC, negative control.

invasion in C-33A cells (Fig. 3E) suggesting that ATG12 may participate in cell metastasis.

Overexpression of miR-378 promotes metastasis of cervical cancer in vivo. To verify the promotion of miR-378 on cervical cancer metastasis in vivo, C-33A cells transfected with GIPZ-miR-378 or negative control were injected into mice via a tail vein injection. Following 8 weeks incubation, lungs and tumor foci were compared between the GIPZ-miR-378 and control groups. Histological analysis of the lungs revealed that miR-378 overexpression promoted lung metastasis (Fig. 4A). Lungs with fewer metastasis nodules were observed in the control group than the GIPZ-miR-378 group (Fig. 4B). In addition, the number of tumor foci was significantly increased in the GIPZ-miR-378 group when compared with in the control group (Fig. 4C).

\section{Discussion}

In recent years, miRNAs have been reported to be involved in tumor initiation, promotion and progression (6). In the present study, miR-378 was observed to be frequently upregulated in CIN III and cervical cancer tissues when compared with normal cervix tissues, and overexpression of miR-378 significantly promoted cell metastasis in vitro and in vivo, while the inhibition of miR-378 significantly decreased tumor metastasis in vitro; ATG12 was identified as a functional and direct target of miR-378 in the present study.

miR-378 has been identified to be aberrantly expressed in a number of types of cancer and may be applied as an early diagnostic biomarker (14). In gastric cancer, miR-378 functions as a tumor suppressor by inhibiting tumor growth via the suppression of cyclin dependent kinase (CDK) 6 and vascular epithelial growth factor signaling (15). In colorectal cancer, miR-378 inhibited tumor growth and invasion (16), and the plasma levels of miR-378 may be used as a diagnostic biomarker to discriminate colorectal cancer patients from healthy individuals (16). Downregulated miR-378 correlated with tumor invasiveness and poor prognosis of patients with glioma (17). In addition to functioning as a tumor suppressor, miR-378 also acts as an onco-miRNA. In liver cancer, miR-378 enhanced cell survival, tumor growth and metastasis by downregulating suppressor of fused homolog and FUS RNA binding protein expression $(18,19)$. Upregulation of miR-378 markedly promoted cell proliferation, colony formation, migration and invasion in vitro, as well as tumor growth in vivo by downregulating transducer of receptor tyrosine-protein kinase erbB-2 (TOB2) (20). The increased serum levels of miR-378 served as powerful non-invasive diagnostic and prognostic biomarkers in renal cell carcinoma (21). In cervical cancer, Wang et al (4) demonstrated that miR-378 expression levels were significantly increased in HPV-infected tissues, which was consistent with the findings in the present study, that miR-378 was significantly upregulated in CIN III and cervical cancers. In the present study, miR-378 was demonstrated to serve as an onco-RNA in cervical cancer as the overexpression of miR-378 promoted cell migration and invasion in vitro. To the best of our knowledge, the present study is the first to investigate the functional role of miR-378 in the migration and invasion of cervical cancer. 
It has been reported that miR-378 is a direct target of the c-Myc oncoprotein, which functions as a transcription factor that serves as a master regulator in various biological processes (22). However, a potential association between miR-378 and autophagy was revealed as ATG12 was identified to be a direct target of miR-378 as determined by a luciferase reporter assay in the present study. It is generally accepted that ATG12 is an important factor in apoptosis vacuole formation (23) by conjugating to ATG5 or ATG3. ATG12 induces mitophagy, mitochondrial fusion and activates mitochondrial apoptosis by conjugating to ATG5, and promotes mitochondrial fusion and restricts mitochondrial mass by conjugating to ATG3 (11). Free ATG12 promotes mitochondrial apoptosis in a similar manner to proapoptotic B-cell lymphoma-2 homology 3-only proteins (24). Studies have demonstrated that downregulation of ATG12 promoted tumor growth by inhibiting cell autophagy $(25,26)$. In the present study, the overexpression of miR-378 reduced ATG12 expression in C-33A cells, and miR-378 inhibitor transfection increased ATG12 expression in HeLa cells. In addition to ATG12,CCND1 and pRb were also downregulated by miR-378. The CCND1 gene encodes the cyclin D1 protein, which is required for progression through the G1 phase of the cell cycle. It was demonstrated that the Myc-miR-378-transducer of Erb-B receptor kinase 2, 2 (TOB2) signaling pathway converges on the CCND1 level to promote transformation, and TOB2 acts to inhibit CCND1 expression (27). pRb, which controls the G1-S checkpoint of the cell cycle, was inhibited by the CCND1-CDK4 complex in the promotion of passage via the G1 phase. It has been reported that in gastric cancer, aberrantly expressed $\mathrm{pRb}$ and CCND1 were associated with lymph node metastases (28). Thus, miR-378 may modulate cervical cancer metastasis by regulating ATG12, CCND1 and pRb.

In conclusion, the present study demonstrated that miR-378 significantly promoted cervical cancer metastasis in vitro and in vivo. ATG12 was identified as a direct and functional target of miR-378. The findings of the present study suggested that miR-378 may provide novel insights into the molecular mechanism of the pathology and therapeutic targets in cervical cancer patients.

\section{Acknowledgements}

Not applicable.

\section{Funding}

The present study was conducted at Qilu Hospital, Shandong University and was supported by the National Natural Science Foundation of China (grant no. 81572559), The Key Research Project of Shandong Province (grant no. 2017CXGC1210), The Science and Technology Development Plan of Shandong Province (grant no. 2014GH218029) and the National Science and Technology Project of China (grant no. 2015BAI13B05).

\section{Availability of data and materials}

The analyzed data sets generated during the study are available from the corresponding author on reasonable request.

\section{Authors' contributions}

DT, CZ and SH designed the study and performed the experiments. XH and SK performed the statistical analysis and $\mathrm{YZ}$ was involved in patient recruitment. All authors reviewed and approved the final manuscript.

\section{Ethics approval and consent to participate}

Written informed consent was obtained from all patients and the present study was approved by the Ethics Committee of Qilu Hospital of Shandong University (Jinan, China).

All animal experiments were approved by the Ethics Committee of Qilu Hospital of Shandong University (Jinan, China).

\section{Consent for publication}

Written informed consent was obtained from all patients.

\section{Competing interests}

The authors declare that they have no competing interests.

\section{References}

1. Siegel RL, Miller KD and Jemal A: Cancer statistics, 2015. CA Cancer J Clin 65: 5-29, 2015.

2. Muñoz N, Bosch FX, de Sanjosé S, Herrero R, Castellsagué X, Shah KV, Snijders PJ and Meijer CJ; International Agency for Research on Cancer Multicenter Cervical Cancer Study Group: Epidemiologic classification of human papillomavirus types associated with cervical cancer. N Engl J Med 348: 518-527, 2003.

3. Sakuragi N: Up-to-date management of lymph node metastasis and the role of tailored lymphadenectomy in cervical cancer. Int J Clin Oncol 12: 165-175, 2007.

4. Wang X, Wang HK, Li Y, Hafner M, Banerjee NS, Tang S, Briskin D, Meyers C, Chow LT, Xie X, et al: microRNAs are biomarkers of oncogenic human papillomavirus infections. Proc Natl Acad Sci USA 111: 4262-4267, 2014.

5. Tsujiura M, Ichikawa D, Komatsu S, Shiozaki A, Takeshita H, Kosuga T, Konishi H, Morimura R, Deguchi K, Fujiwara H, et al: Circulating microRNAs in plasma of patients with gastric cancers. Br J Cancer 102: 1174-1179, 2010.

6. Chen X, Ba Y, Ma L, Cai X, Yin Y, Wang K, Guo J, Zhang Y, Chen J, Guo X, et al: Characterization of microRNAs in serum: A novel class of biomarkers for diagnosis of cancer and other diseases. Cell Res 18: 997-1006, 2008.

7. Heneghan HM, Miller N, Lowery AJ, Sweeney KJ, Newell J and Kerin MJ: Circulating microRNAs as novel minimally invasive biomarkers for breast cancer. Ann Surg 251: 499-505, 2010.

8. Wen SY, Lin Y, Yu YQ, Cao SJ, Zhang R, Yang XM, Li J, Zhang YL, Wang YH, Ma MZ, et al: miR-506 acts as a tumor suppressor by directly targeting the hedgehog pathway transcription factor Gli3 in human cervical cancer. Oncogene 34: 717-725, 2015.

9. Li BH, Zhou JS, Ye F, Cheng XD, Zhou CY, Lu WG and Xie X: Reduced miR-100 expression in cervical cancer and precursors and its carcinogenic effect through targeting PLK1 protein. Eur J Cancer 47: 2166-2174, 2011.

10. Xu J, Li Y, Wang F, Wang X, Cheng B, Ye F, Xie X, Zhou C and Lu W: Suppressed miR-424 expression via upregulation of target gene Chk1 contributes to the progression of cervical cancer. Oncogene 32: 976-987, 2013.

11. Haller M, Hock AK, Giampazolias E, Oberst A, Green DR, Debnath J, Ryan KM, Vousden KH and Tait SW: Ubiquitination and proteasomal degradation of ATG12 regulates its proapoptotic activity. Autophagy 10: 2269-2278, 2014.

12. Murrow L, Malhotra R and Debnath J: ATG12-ATG3 interacts with Alix to promote basal autophagic flux and late endosome function. Nat Cell Biol 17: 300-310, 2015. 
13. Livak KJ and Schmittgen TD: Analysis of relative gene expression data using real-time quantitative PCR and the 2(-Delta Delta C(T)) method. Methods 25: 402-408, 2001.

14. Li ZZ, Shen LF, Li YY, Chen P and Chen LZ: Clinical utility of microRNA-378 as early diagnostic biomarker of human cancers: A meta-analysis of diagnostic test. Oncotarget 7: 58569-58578, 2016.

15. Deng H, Guo Y, Song H, Xiao B, Sun W, Liu Z, Yu X, Xia T, Cui L and Guo J: MicroRNA-195 and microRNA-378 mediate tumor growth suppression by epigenetical regulation in gastric cancer. Gene 518: 351-359, 2013.

16. Zanutto S, Pizzamiglio S, Ghilotti M, Bertan C, Ravagnani F Perrone F, Leo E, Pilotti S, Verderio P, Gariboldi $M$ and Pierotti MA: Circulating miR-378 in plasma: A reliable, haemolysis-independent biomarker for colorectal cancer. Br J Cancer 110: 1001-1007, 2014.

17. Li B, Wang Y, Li S, He H, Sun F, Wang C, Lu Y, Wang X and Tao B: Decreased expression of miR-378 correlates with tumor invasiveness and poor prognosis of patients with glioma. Int J Clin Exp Pathol 8: 7016-7021, 2015.

18. Lee DY, Deng Z, Wang CH and Yang BB: MicroRNA-378 promotes cell survival, tumor growth, and angiogenesis by targeting $\mathrm{SuFu}$ and Fus-1 expression. Proc Natl Acad Sci USA 104: 20350-20355, 2007.

19. Ma J, Lin J, Qian J, Qian W, Yin J, Yang B, Tang Q, Chen X, Wen X, Guo H and Deng Z: MiR-378 promotes the migration of liver cancer cells by down-regulating Fus expression. Cell Physiol Biochem 34: 2266-2274, 2014

20. Yu BL, Peng XH, Zhao FP, Liu X, Lu J, Wang L, Li G, Chen HH and Li XP: MicroRNA-378 functions as an onco-miR in nasopharyngeal carcinoma by repressing TOB 2 expression. Int J Oncol 44: 1215-1222, 2014.

21. Fedorko M, Stanik M, Iliev R, Redova-Lojova M, Machackova T, Svoboda M, Pacik D, Dolezel J and Slaby O: Combination of MiR-378 and MiR-210 serum levels enables sensitive detection of renal cell carcinoma. Int J Mol Sci 16: 23382-23389, 2015.
22. Dang CV: c-Myc target genes involved in cell growth, apoptosis, and metabolism. Mol Cell Biol 19: 1-11, 1999.

23. Metlagel Z, Otomo C, Takaesu G and Otomo T: Structural basis of ATG3 recognition by the autophagic ubiquitin-like protein ATG12. Proc Natl Acad Sci USA 110: 18844-18849, 2013.

24. Rubinstein AD, Eisenstein M, Ber Y, Bialik S and Kimchi A The autophagy protein Atg12 associates with antiapoptotic Bcl-2 family members to promote mitochondrial apoptosis. Mol Cell 44: 698-709, 2011.

25. Cordani M, Oppici E, Dando I, Butturini E, Dalla Pozza E, Nadal-Serrano M, Oliver J, Roca P, Mariotto S, Cellini B, et al: Mutant p53 proteins counteract autophagic mechanism sensitizing cancer cells to mTOR inhibition. Mol Oncol 10: 1008-1029, 2016.

26. Pan B, Feng B, Chen Y, Huang G, Wang R, Chen L and Song H: MiR-200b regulates autophagy associated with chemoresistance in human lung adenocarcinoma. Oncotarget 6: 32805-32820, 2015.

27. Feng M, Li Z, Aau M, Wong $\mathrm{CH}$, Yang $\mathrm{X}$ and $\mathrm{Yu} \mathrm{Q}$ : Myc/miR-378/TOB2/cyclin D1 functional module regulates oncogenic transformation. Oncogene 30: 2242-2251, 2011.

28. Feakins RM, Nickols CD, Bidd H and Walton SJ: Abnormal expression of $\mathrm{pRb}, \mathrm{p} 16$, and cyclin $\mathrm{D} 1$ in gastric adenocarcinoma and its lymph node metastases: Relationship with pathological features and survival. Hum Pathol 34: 1276-1282, 2003

(c) (1) () This work is licensed under a Creative Commons Attribution-NonCommercial-NoDerivatives 4.0 International (CC BY-NC-ND 4.0) License. 